\title{
Scabies escaping detection until dermoscopy was applied
}

\author{
Aimilios Lallas ${ }^{1}$, Zoe Apalla ${ }^{1}$, Elizabeth Lazaridou ${ }^{1}$, Elena Sotiriou ${ }^{1}$, Efstratios Vakirlis ${ }^{1}$, \\ Dimitrios Ioannides ${ }^{1}$
}

1 First Department of Dermatology, Aristotle University, Thessaloniki, Greece

Citation: Lallas A, Apalla Z, Lazaridou E, Sotiriou E, Vakirlis E, Ioannides D. Scabies escaping detection until dermoscopy was applied. Dermatol Pract Concept. 2017;7(1):9. DOI: https://doi.org/10.5826/dpc.0701a09

Received: November 13, 2016; Accepted: November 26, 2016; Published: January 31, 2017

Copyright: $@ 2017$ Lallas et al. This is an open-access article distributed under the terms of the Creative Commons Attribution License, which permits unrestricted use, distribution, and reproduction in any medium, provided the original author and source are credited.

Funding: None.

Competing interests: The authors have no conflicts of interest to disclose.

All authors have contributed significantly to this publication.

Corresponding author: Aimilios Lallas, MD, MSc, PhD, First Department of Dermatology, Aristotle University, Thessaloniki, Greece, 124 Delfon Str, 54643, Thessaloniki, Greece. Tel. 00302313308882; Fax.00302310277979. Email: emlallas@gmail.com

\begin{abstract}
Dermoscopy is already considered a fairly established method for diagnosing scabies. This is because dermoscopy enables the visualization both of the burrow and the mite itself, forming the so-called "jet with a contrail” structure. In the present report we present an extraordinary case of a patient with scabies lesions on the face and neck, which was misdiagnosed during sequential visits and underwent unnecessary surgical diagnostic procedures. Finally, the diagnostic problem was solved when dermoscopy was applied.
\end{abstract}

\section{Case Presentation}

After repeated previous consultations, a 73-year-old woman suffering for several months from itchy plaques and nodules on the face and neck presented for her follow-up visit (Figures 1,2). A biopsy had been performed during previous consultations and the histopathologic diagnosis lay between lymphoma and pseudo-lymphoma. The subsequent immunohistochemical assay did not solve the diagnostic problem. Dermoscopy of the lesions revealed a perfectly demarcated burrow and, at its end, a scabies mite (Figure 3). The diagnosis of scabies was straightforward and confirmed microscopically after scraping test. The eruption remitted completely after appropriate treatment for scabies.

\section{Discussion}

The accuracy of dermoscopy for the diagnosis of scabies has been assessed as at least equal to microscopic examination after skin scraping [1,2]. In fact, dermoscopy has the advantage of rapidly screening several lesions of the patient, in contrast to the microscopic examination which is performed by scraping only one or two lesions. Given that in otherwise healthy individuals, no more that 4-5 mites exist on the skin at on time point, scanning all the lesions of the patient minimizes the possibility of a false negative result.

In our patient, the atypical clinical manifestation and, especially, the distribution of the eruption on the face, misled the clinicians on repeated visits, whereas dermoscopy was not 


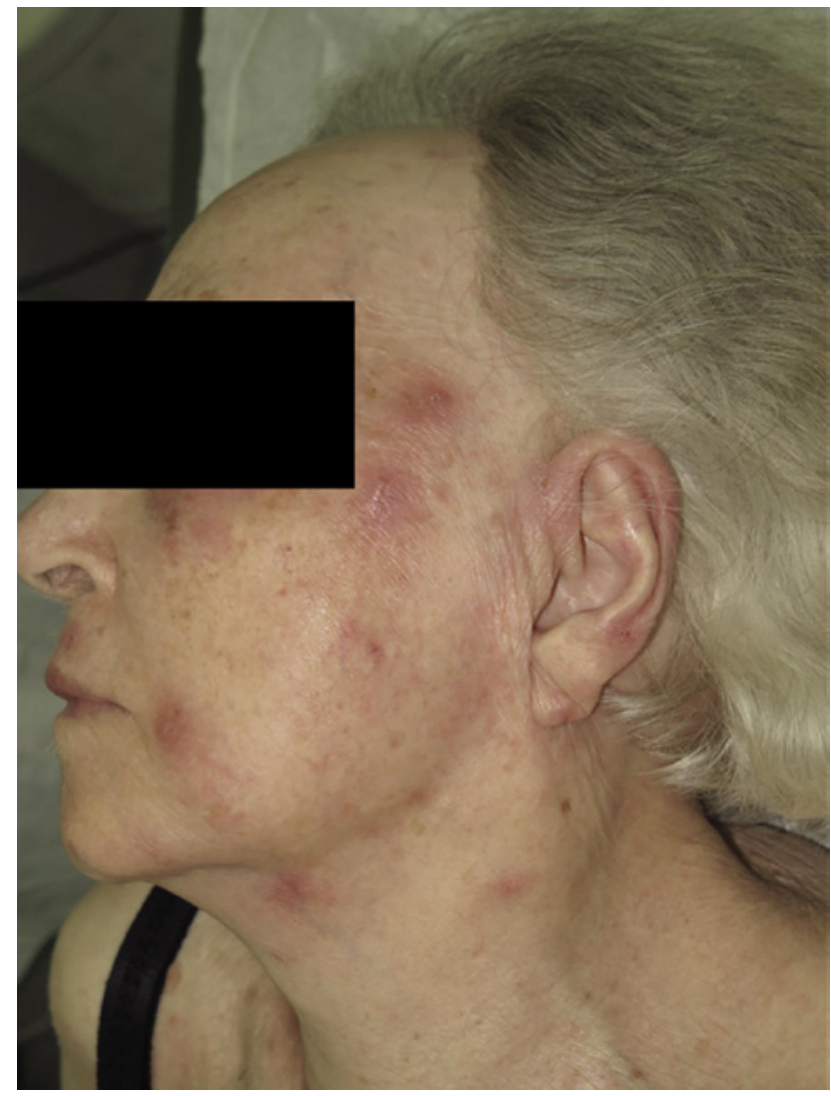

Figure 1. A 6-month-old standing pruritic eruption on the face and neck of a 73-year-old woman. [Copyright: @2017 Lallas et al.]

performed during the initial visits, because scabies was not even included in the differential diagnosis. This resulted in unnecessary surgical procedures and histopathologic exams, which might have been continued if dermoscopy was not eventually performed. This case highlights a basic principle of dermoscopy application, namely, that dermoscopy should be applied on every skin lesion and not only in clinically preselected cases [2].

\section{References}

1. Walter B, Heukelbach J, Fengler G, et al. Comparison of dermoscopy, skin scraping, and the adhesive tape test for the diagnosis of scabies in a resource-poor setting. Arch Dermatol. 2011;147:468473.

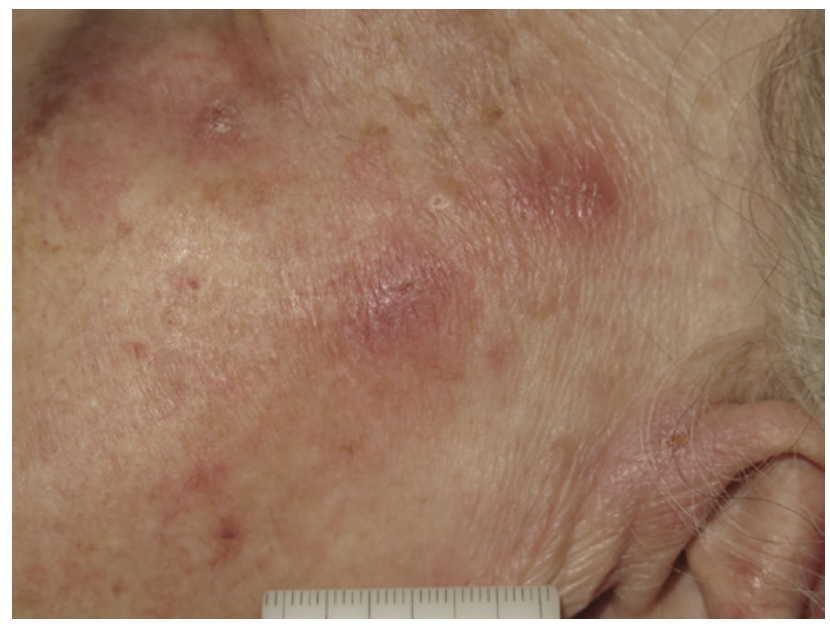

Figure 2. A close-up clinical photo of one of the lesions, highlighting that the eruption consisted of slightly excoriated papules. The clinical differential diagnosis was broad, including discoid lupus erythematosus, sarcoidosis, lymphoma and pseudolymphoma. [Copyright: (C)2017 Lallas et al.]

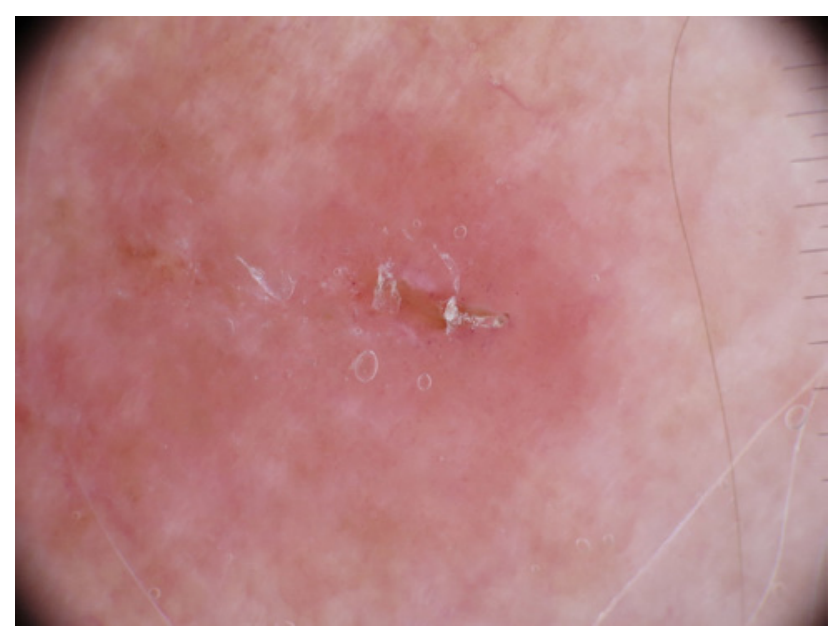

Figure 3. Dermoscopy revealed the typical pattern for scabies (jet with a contrail), consisting of an evident burrow ending with a triangular brownish projection, corresponding to the mite. The eruption remitted completely after appropriate treatment. [Copyright: @2017 Lallas et al.]

2. Lallas A, Giacomel J, Argenziano G, et al. Dermoscopy in general dermatology: practical tips for the clinician. $\mathrm{Br} J$ Dermatol. 2014;170:514-526. 